\section{De l'importance de la localisation des récepteurs du glutamate NMDA}

${ }^{1}$ Neurocentre Magendie, Inserm U862,

146, rue Léo Saignat, 33077 Bordeaux, France;

${ }^{2}$ Université de Bordeaux, Bordeaux, France ;

${ }^{3}$ Department of neuroscience,

Tufts university school of medicine, Boston, États-Unis.

stephane.oliet@inserm.fr
> Les récepteurs du glutamate de type NMDA (acide $\mathrm{N}$-méthyl-D-aspartique) jouent un rôle primordial dans le système nerveux des mammifères. Ils sont à la base des phénomènes de plasticité synaptique les plus répandus : la potentialisation à long terme (LTP) et la dépression à long terme (LTD), qui représentent les mécanismes cellulaires de la mémoire et de l'apprentissage [1]. Les récepteurs du glutamate de type NMDA interviennent également dans le phénomène dit d'excitotoxicité, responsable de la mort des neurones au cours des maladies neurodégénératives comme la maladie d'Alzheimer et la sclérose latérale amyotrophique. Au vu de ces fonctions majeures, mais opposées, auxquelles ils contribuent, ces récepteurs ont été étudiés en détail par de très nombreux laboratoires dans le monde entier, à l'aide de techniques et modèles très variés.

\section{Les différents ligands des récepteurs} de type NMDA

Ainsi, il a été découvert il y a 25 ans que, contrairement à la plupart des autres récepteurs de neurotransmetteurs, les récepteurs du glutamate de type NMDA nécessitent, non pas un, mais deux agonistes pour être activés. Le premier est le glutamate, principal neurotransmetteur excitateur du cerveau, qui est libéré par les synapses lorsque deux neurones échangent des informations excitatrices. Le second est qualifié de coagoniste, et fut identifié comme étant la glycine. Le site de liaison qui permet au récepteur de type NMDA de reconnaître et fixer la glycine a été baptisé site glycine [2]. Pourtant, au cours des dernières années, de nombreux travaux, y compris ceux de notre équipe, ont permis d'identifier un autre composé endogène capable de se fixer sur le même site et de jouer le rôle de coagoniste de ces récepteurs NMDA : la D-sérine [3]. Cette substance est produite et libérée par les cellules astrogliales en contact avec les synapses excitatrices, démontrant par là même que ces cellules non neuronales exercent un contrôle étroit sur l'activité des récepteurs NMDA synaptiques et les fonctions qui en découlent [4-6]. II s'avère que les récepteurs NMDA sont présents non seulement au niveau des synapses, mais également à l'extérieur de cellesci (récepteurs extrasynaptiques). Si les recherches concernant l'identité du coagoniste des récepteurs NMDA synaptiques ont été très actives, aucune n'a cherché à identifier le coagoniste des récepteurs NMDA extrasynaptiques. Ces récepteurs extrasynaptiques intéressent pourtant grandement la communauté neuroscientifique, et constituent une cible thérapeutique jugée prometteuse. $\varepsilon n$ effet, ils semblent spécifiquement responsables des effets délétères associés à l'excitotoxicité résultant de leur activation, tandis que les effets bénéfiques (neuroprotection et plasticité) seraient préférentiellement associés aux récepteurs synaptiques [7].

\section{Rôle de la glycine et de la D-sérine} dans l'activation des récepteurs NMDA Dans notre dernière publication [8], nous nous sommes intéressés au rôle respectif de la glycine et de la D-sérine dans l'activité des récepteurs NMDA synaptiques et extrasynaptiques, puis à la contribution de ces deux populations de récepteurs à différents processus physiologiques et pathologiques. Nos travaux révèlent (1) que les récepteurs NMDA utilisent un coagoniste différent en fonction de leur localisation, et (2) que les deux types de récepteurs, synaptiques et extrasynaptiques, jouent des rôles bien distincts, quoiqu'inattendus, dans la plasticité synaptique et l'excitotoxicité neuronale.

Pour cette étude, nous nous sommes intéressés aux synapses que forment les collatérales de Schaffer (ou axones collatéraux des neurones pyramidaux de CA3) sur les neurones pyramidaux CAl de l'hippocampe chez le rat adulte. L'hippocampe est une région cérébrale directement associée à la mémoire, dans laquelle les récepteurs NMDA, ainsi que la plasticité à long terme et les phénomènes d'excitotoxicité, ont été très largement étudiés ; elle est particulièrement affectée dans la maladie d'Alzheimer. Nous avons effectué des enregistrements électrophysiologiques sur des tranches de cerveau dans lesquelles nous avons fait varier les taux de D-sérine et de glycine par des traitements enzymatiques. Ainsi, nous avons démontré que l'activité des récepteurs NMDA synaptiques est bien gouvernée par la présence de D-sérine, alors qu'elle n'est pas sensible à la glycine endogène. Par ailleurs, en raison de la présence d'un transporteur très efficace de type GlyT-1 sur les cellules astrogliales, des quantités insignifiantes de glycine accèdent à la synapse. Seule 


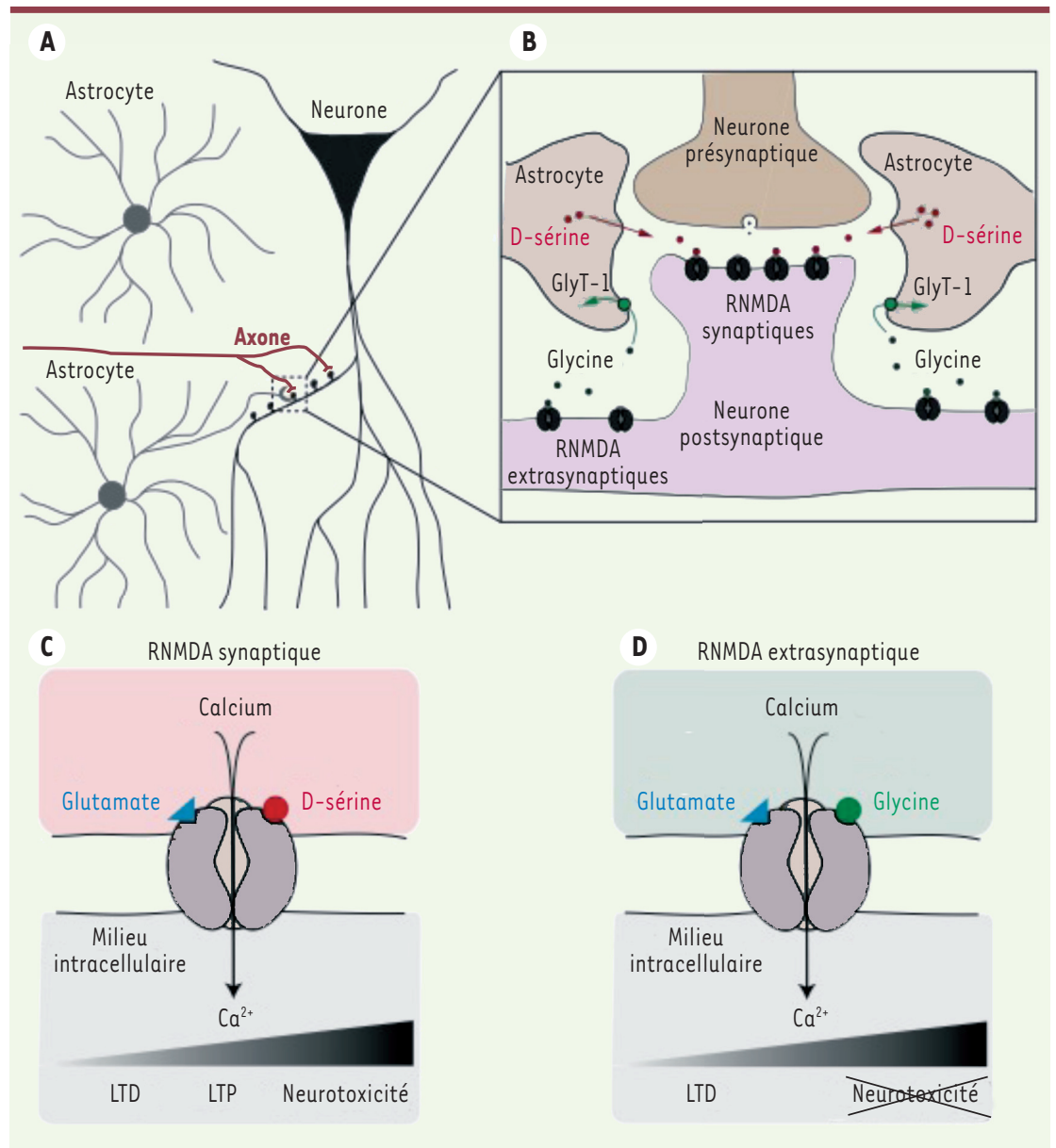

Figure 1. Fonctionnement des récepteurs NMDA synaptiques et extrasynaptiques. A. Les axones provenant des neurones de la région CA3 (en marron) forment des synapses avec les dendrites des neurones de la région CAl (en noir). Les fins prolongements émis par les astrocytes (gris) contactent et enrobent ces synapses. B. Illustration des récepteurs NMDA synaptiques et extrasynaptiques et de leurs coagonistes endogènes. Tandis que la glycine (en vert) est disponible pour les récepteurs NMDA extrasynaptiques, l'activité des transporteurs de la glycine GlyT-1 localisés sur les prolongements des astrocytes au voisinage de la synapse empêche ce coagoniste d'accéder à la synapse. Les récepteurs NMDA synaptiques utilisent comme coagoniste la D-sérine (en rouge) qui est libérée dans la synapse par les astrocytes. C. L'activité des récepteurs NMDA synaptiques est donc gouvernée par la D-sérine. Le couple récepteur NMDA synaptique/D-sérine est impliqué dans la potentialisation (LTP) et la dépression (LTD) synaptiques à long terme, qui sont les deux formes de plasticité synaptique les plus courantes dans le système nerveux central. Ces récepteurs sont également impliqués dans la mort neuronale par neurotoxicité, lorsqu'ils sont activés de manière trop intense. $D$. Les récepteurs NMDA extrasynaptiques, dont l'activité est contrôlée par la glycine, ne semblent pas être impliqués dans la mort neuronale induite par une activation massive et prolongée des récepteurs NMDA (neurotoxicité), ni jouer aucun rôle dans l'induction de la LTP. Ils s'avèrent, en revanche, tout aussi essentiels que leurs homologues synaptiques pour la LTD.

l'inhibition pharmacologique des transporteurs GlyT-l exprimés par les cellules astrogliales voisines permet à la glycine endogène d'accéder à la synapse et de participer à l'activité des récepteurs pas de la D-sérine. Ce résultat est cohérent avec la présence en quantité abondante de la glycine dans l'espace extrasynaptique, tandis que la D-sérine est probablement libérée à proximité des contacts synaptiques, de telle sorte que sa concentration n'atteint pas des niveaux suffisants pour jouer un rôle au niveau des récepteurs NMDA en dehors de la synapse (Figure 1).

\section{Récepteurs NMDA synaptiques} et extrasynaptiques : rôles respectifs dans la LTP et l'excitotoxicité

Sur la base de cette dichotomie marquante entre récepteurs NMDA synaptiques et extrasynaptiques, nous avons poursuivi notre étude en examinant les rôles respectifs de ces deux populations de récepteurs dans les processus de plasticité à long terme et d'excitotoxicité (Figure 1). Nous montrons d'abord que la LTP induite au niveau des synapses CA3-CAl n'est pas affectée lorsque l'on inhibe l'activité des récepteurs extrasynaptiques de manière expérimentale, alors qu'elle diminue beaucoup lorsque ce sont les récepteurs NMDA synaptiques qui sont ciblés. De manière surprenante, l'inhibition des récepteurs NMDA, qu'ils soient synaptiques ou extrasynaptiques, empêche l'induction de LTD, suggérant que les deux populations de récepteurs NMDA sont nécessaires à I'induction de cette forme de plasticité. Finalement, dans la dernière partie de notre étude, nous nous sommes intéressés à la mort neuronale induite pharmacologiquement par une activation prolongée de tous les récepteurs NMDA. Ce type de manipulation provoque une entrée massive de $\mathrm{Ca}^{2+}$ dans les neurones, ce qui entraîne leur mort (excitotoxicité). Nous montrons que l'inhibition des récepteurs extrasynaptiques ne protège en rien les neurones d'une telle mort cellulaire, tandis que l'inhibition des récepteurs synaptiques confère une forte résistance cellulaire à cette condition toxique. Ces résultats sont d'autant plus intéressants qu'ils 
contredisent plusieurs études antérieures suggérant que l'excitotoxicité implique les récepteurs NMDA extrasynaptiques dans des préparations de neurones en culture [7]. Cependant, ils sont en parfait accord avec d'autres travaux, similaires aux nôtres, qui démontrent que dégrader la $D$-sérine dans des tranches de cerveaux procure un effet neuroprotecteur [9]. Ainsi, il semble que le phénomène d'excitotoxicité soit régulé par des mécanismes différents dans des préparations de neurones en culture et dans des tranches de cerveaux de rongeurs adultes, deux modèles dans lesquels la distribution de la D-sérine et de la glycine et les fonctions jouées par les récepteurs NMDA synaptiques et extrasynaptiques ne sont probablement pas les mêmes.

\section{Conclusion}

$\varepsilon$ n résumé, nos résultats révèlent deux aspects novateurs de l'activité des récepteurs NMDA. Le premier concerne leur mode opératoire : le coagoniste endogène qui gouverne leur activité diffère selon la localisation cellulaire des récepteurs, de sorte que deux populations fonctionnellement distinctes semblent exister : les récepteurs synaptiques, contrôlés par la D-sérine, et les récepteurs extrasynaptiques, contrôlés par la glycine. Le second aspect concerne le rôle de ces deux populations de récepteurs dans la plasticité synaptique et l'excitotoxicité. Alors que les récepteurs synaptiques sont suffisants et nécessaires pour induire l'excitotoxicité et la LTP, la LTD, en revanche, requiert l'activation conjointe des deux populations de récepteurs. En outre, nos travaux remettent en question la proposition de considérer les récepteurs extrasynaptiques comme une cible thérapeutique dans les maladies neurodégénératives. Ils ouvrent en revanche de nouvelles perspectives quant aux rôles des récepteurs synaptiques et leur régulation par la D-sérine d'origine gliale dans ces pathologies. $\diamond$

Importance of the location

of NMDA glutamate receptors

\section{LIENS D'INTÉRÊT}

Les auteurs déclarent n'avoir aucun lien d'intérêt concernant les données publiées dans cet article.

\section{RÉFÉRENCES}

1. Malenka RC, Bear MF. LTP and LTD: an embarrassment of riches. Neuron $2004 ; 44: 5-21$.

2. Johnson JW, Ascher P. Glycine potentiates the NMDA response in cultured mouse brain neurons. Nature $1987: 325: 529-31$.

3. Mothet JP, Parent AT, Wolosker H, et al. D-serine is an endogenous ligand for the glycine site of the $\mathrm{N}$-methyl-D-aspartate receptor. Proc Natl Acad Sci USA $2000 ; 97:$ : 4926-31.

4. Mothet JP, Pollegioni L, Ouanounou G, et al. Glutamate receptor activation triggers a calcium-dependent and SNARE protein-dependent release of the gliotransmitter D-serine. Proc Natl Acad Sci USA 2005 ; 102 : 5606-11.

5. Panatier A, Theodosis DT, Mothet JP, et al. Gliaderived $D$-serine controls NMDA receptor activity and synaptic memory. Cell 2006 ; $125: 775-84$.

6. Henneberger C, Papouin T, Oliet SH, Rusakov DA. Longterm potentiation depends on release of $D$-serine from astrocytes. Nature $2010 ; 463: 232-6$.

7. Hardingham GE, Bading H. Synaptic versus extrasynaptic NMDA receptor signalling: implications for neurodegenerative disorders. Nat Rev Neurosci $2010 ; 11: 682-96$.

8. Papouin T, Ladépêche L, Ruel J, et al. Synaptic and extrasynaptic NMDA receptors are gated by different endogenous coagonists. Cell 2012 ; 150 : 633-46.

9. Katsuki H, Nonaka M, Shirakawa H, et al. Endogenous $\mathrm{D}$-serine is involved in induction of neuronal death by $\mathrm{N}$-methyl-D-aspartate and simulated ischemia in rat cerebrocortical slices.J Pharmacol Exp Ther 2004 ; $311: 836-44$

\section{NOUVELLE}

\section{Production d'un pancréas fonctionnel in vivo par complémentation de blastocyste} Preuve de concept chez le porc

Laure Coulombel médecine/sciences, ADR Inserm Paris V, 2, rue d'Alésia, 75014 Paris, France.

laure.coulombel@inserm.fr
> Nous avons relaté il y a quelques mois dans une Nouvelle les résultats de la complémentation de blastocystes de souris $p d x 1^{-/-}$(un gène clé de la spécification du pancréas) par des iPS (induced pluripotent stem cells) de rat. Après leur transfert dans un utérus de souris, certains de ces embryons chimères interespèces ont abouti à la naissance de souris dont le pancréas, tout à fait fonctionnel, était entièrement dérivé des iPS de rat [1]. Il y avait donc deux résultats: la création d'une chimère interespèce (souris/rat), et la restauration d'un organe complet, le pancréas, chez l'espèce qui en était dépourvue. Il était clair que l'équipe de $H$. Nakauchi ne s'arrêterait pas là. La seconde étape, l'application de cette démarche à un gros animal, le porc, vient d'être publiée dans Proceedings of the National Academy of Sciences of USA [2].

\section{Acte 1. Créer des fœtus de porcs sans pancréas}

Le développement du pancréas a été bloqué in vivo chez les porcs par la surexpression d'un transgène, Hesl (hairy 